\title{
Downlink Resource Reuse for Device-to-Device Communications Underlaying Cellular Networks
}

\author{
Daohua Zhu, Student Member, IEEE, Jiaheng Wang, Member, IEEE, A. Lee Swindlehurst, Fellow, IEEE, and \\ Chunming Zhao, Member, IEEE
}

\begin{abstract}
The full potential of Device-to-device (D2D) communication relies on efficient resource reuse strategies including power control and matching of D2D links and cellular users (CUs). This letter investigates downlink resource reuse between multiple D2D links and multiple CUs. Our goal is to achieve a network utility enhancement for D2D communication while ensuring the QoS of the CUs. Despite the combinatorial nature of the problem and the coupled power constraints, we characterize the optimal D2D-CU matching as well as their power coordination, and propose an efficient algorithm to jointly optimize all D2D links and CUs. The proposed downlink resource reuse strategy shows a superiority over existing D2D schemes.
\end{abstract}

Index Terms-Device-to-device communication, joint optimization, quality of service, resource reuse strategy.

\section{INTRODUCTION}

D EVICE-TO-DEVICE communication is a promising technique to improve spectral utilization by supporting direct communication between two closely located users which would otherwise have to be relayed by a base station (BS) in traditional cellular systems. In addition to high spectral efficiency, D2D communication also enjoys several advantages in terms of data rate, latency and power consumption, etc. It is worth noting that the advantage of $\mathrm{D} 2 \mathrm{D}$ communication in enhancing physical layer security has also been investigated in [1]. Therefore, D2D techniques have been proposed to be incorporated into current and future wireless networks [2].

In cellular systems, D2D links may reuse either uplink or downlink resources of CUs for direct communications. Flexible and efficient resource reuse (RR) schemes are crucial for

Manuscript received January 05, 2014; accepted February 22, 2014. Date of publication February 28, 2014; date of current version March 06, 2014. This work was supported by the 973 Program (2013CB336600 and 2013CB329203), National Natural Science Foundation of China (Grant 61201174), the Natural Science Foundation of Jiangsu (Grant BK2012325), the Fundamental Research Funds for the Central Universities, and the China Scholarship Council. The associate editor coordinating the review of this manuscript and approving it for publication was Prof. Xin Wang.

D. Zhu is with the National Mobile Communications Research Laboratory, Southeast University, Nanjing 210096, China, on leave as a visiting student in the Department of Electrical Engineering and Computer Science, University of California, Irvine, CA 92697-2625 USA (email: zhudaohua@seu.edu.cn).

J. Wang and C. Zhao are with the National Mobile Communications Research Laboratory, Southeast University, Nanjing 210096, China (email: jhwang@seu. edu.cn; cmzhao@seu.edu.cn).

A. L. Swindlehurst is with Center for Pervasive Communications and Computing, University of California, Irvine, CA 92697-2625 USA (email: swindle@uci.edu).

Color versions of one or more of the figures in this paper are available online at http://ieeexplore.ieee.org.

Digital Object Identifier 10.1109/LSP.2014.2309143 underlay D2D communication and have thus received much attention [3]-[6]. In particular, [3] studied the sum rate maximization of one D2D link and one CU, while [4] proposed an interference control scheme for one D2D user with multiple CUs. The RR problems for multiple D2D links and CUs were considered in [5], [6], but in these papers each D2D link can only reuse one $\mathrm{CU}$ resource.

Most existing works have (e.g. [3]-[5]) considered uplink resource reuse for the sake of asymmetric uplink and downlink service loads. However, the near-far effect may cause strong interference to CUs when D2D links are close to a BS. Therefore, downlink resource reuse is also very important. D2D communication is basically a complement to traditional cellular communication. Hence, CUs generally have higher priority and their QoS must be properly protected [2], [3].

We consider multiple D2D links that reuse the downlink resources of multiple CUs in a cellular network. We formulate the RR design as a generic utility maximization problem for the D2D links with QoS constraints for the CUs. Each D2D link is allowed to reuse resources of multiple CUs and thus our result is more general than [5], [6]. Our goal is to jointly optimize the D2D-CU matching and power control of both D2D links and CUs so that the network utility is maximized and the service quality of CUs is protected.

The formulated RR problem with QoS constraints is a mixed integer program and is not convex. The power constraint at the BS also presents another challenge in contrast with the uplink RR designs in [3]-[5]. We overcome these difficulties by transforming the original problem into a more tractable form, based on which we characterize the optimal power control and D2D-CU matching. Then, we propose an efficient algorithm to jointly optimize the D2D links and CUs, and achieve the optimal RR solution. The superiority of the proposed RR scheme is verified by numerical results.

\section{System Model And Problem Statement}

Consider a hybrid single-cell network as shown in Fig. 1, consisting of $C$ orthogonal downlink CUs (each occupies a dedicated resource block (RB)) and $L$ D2D links. Let $y_{l, c} \in\{0,1\}$ indicate whether D2D link $l$ reuses the $\mathrm{RB}$ of $\mathrm{CU} c$. To facilitate manipulation and billing of D2D communications, we assume that each CU's RB can be reused by at most one D2D link, i.e., $\sum_{l=1}^{L} y_{l, c} \leq 1, \forall c$. A similar constraint was also adopted in [5] and [6].

We introduce the normalized (by the noise power) gains $g_{c}$, $g_{l, c}, g_{c, l}^{I}$, and $g_{l, c}^{I}$ to represent the transmission channel between the BS and $\mathrm{CU} c$, the transmission channel between the D2D transmitter (TX) and receiver (RX) for D2D link $l$ on the RB 


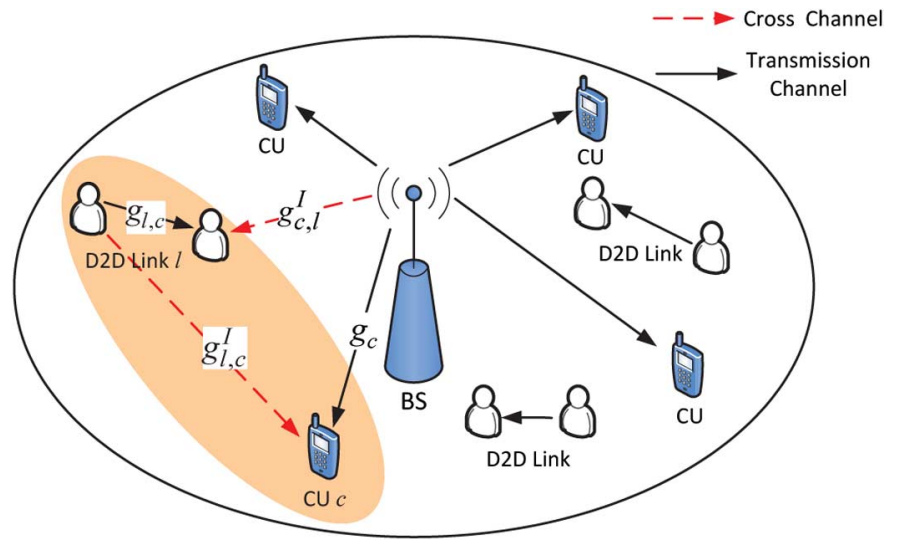

Fig. 1. D2D communications underlaying downlink cellular network and an illustration of interference pattern with one D2D link reusing one CU's RB.

of $\mathrm{CU} c$, the cross channel from the BS to D2D link $l$ on the $\mathrm{RB}$ of $\mathrm{CU} c$, and the cross channel from the D2D TX to CU $c$, respectively. Fig. 1 illustrates such a resource reuse situation and also the associated interference pattern. Hence, the data rate of D2D link $l$ is given by

$$
r_{l}=\sum_{c=1}^{C} y_{l, c} \log _{2}\left(1+\frac{p_{l, c} g_{l, c}}{1+g_{c, l}^{I} p_{c}}\right),
$$

where $p_{l, c} \geq 0$ and $p_{c} \geq 0$ are the transmit power of D2D link $l$ and CU $c$ on the associated RB.

D2D communication is typically implemented as a complement to cellular communication, so CUs generally have higher priority than D2D links. Thus, we impose a QoS target for each $\mathrm{CU}$ to protect the normal cellular communication:

$$
\sum_{l=1}^{L} y_{l, c} \log _{2}\left(1+\frac{p_{c} g_{c}}{1+g_{l, c}^{I} p_{l, c}}\right) \geq R_{c}, \forall c .
$$

Let $U_{l}\left(r_{l}\right)$ be the utility function associated with D2D link $l$. Our goal is to maximize the total utility of the D2D links while satisfying the cellular QoS constraints by properly matching each D2D link with a CU and coordinating their power on the associated RB. Such a design problem can be formulated as

$$
\begin{aligned}
\max _{\left\{y_{l, c}, p_{l, c}, p_{c}\right\}} & \sum_{l=1}^{L} U_{l}\left(r_{l}\right) \\
\text { s.t. } & (2) \\
& \sum_{l=1}^{L} y_{l, c} \leq 1, \forall c \\
& \sum_{c=1}^{C} y_{l, c} p_{l, c} \leq P_{l}^{\max }, \forall l, \\
& \sum_{c=1}^{C} p_{c} \leq P_{B S}^{\max },
\end{aligned}
$$

where each utility $U_{l}\left(r_{l}\right)$ is assumed to be a concave and increasing function, which includes many common system metrics including the sum rate, proportional fairness, etc. as special cases [7]. The BS maintains reliable connections with the CUs under the power budget $P_{B S}^{\max }$. Meanwhile, each D2D link $l$ reuses the cellular RBs under the power budget $P_{l}^{\max }$.

\section{JOINT OPTIMIZATION OF D2D-CU MATCHING AND POWER COORDINATION}

The mixed integer programming and the nonconvexity of the objective function as well as the QoS constraints make the system design in (3) a nontrivial and difficult problem that is intractable in its original form. In this section, we first show that (3) can be equivalently transformed into a more tractable form, as demonstrated in the following proposition.

Proposition 1: Let $d_{l, c} \triangleq g_{c} g_{l, c}, e_{l, c} \triangleq g_{c}+\alpha_{c} g_{c, l}^{I}$, and $f_{l, c} \triangleq \alpha_{c} g_{c, l}^{I} g_{l, c}^{I}$. Then, problem (3) is equivalent to the following optimization problem

$$
\begin{aligned}
\max _{\left\{y_{l, c}, p_{l, c}\right\}} & \sum_{l=1}^{L} U_{l}\left(\tilde{r}_{l}\right) \\
\text { s.t. } & \sum_{l=1}^{L} y_{l, c} \leq 1, \forall c, \\
& \sum_{c=1}^{C} y_{l, c} p_{l, c} \leq P_{l}^{\max }, \forall l, \\
& \sum_{c=1}^{C} \sum_{l=1}^{L} y_{l, c} \frac{\alpha_{c}}{g_{c}} g_{l, c}^{I} p_{l, c} \leq P_{B S}^{\max }-\sum_{c=1}^{C} \frac{\alpha_{c}}{g_{c}},
\end{aligned}
$$

where $\alpha_{c} \triangleq 2^{R_{c}}-1$ and

$$
\tilde{r}_{l}=\sum_{c=1}^{C} y_{l, c} \log _{2}\left[1+d_{l, c} p_{l, c} /\left(e_{l, c}+f_{l, c} p_{l, c}\right)\right] .
$$

Proof: Supposing that D2D link $l$ reuses the $c$ th RB, i.e., $y_{l, c}=1$, then from (2), we have $p_{c} \geq \alpha_{c}\left(1+g_{l, c}^{I} p_{l, c}\right) / g_{c}$. For fixed $p_{l, c}$, the objective function is monotonically decreasing in $p_{c}$, so the optimal $p_{c}$ must be achieved at $p_{c}^{\star}=\alpha_{c}(1+$ $\left.g_{l, c}^{I} p_{l, c}\right) / g_{c}$. Then, the constraint (4d) can be obtained by substituting $p_{c}^{\star}$ into $(3 \mathrm{c})$. Similarly, we can substitute $p_{c}^{\star}$ into the objective function and obtain $\tilde{r}_{l}$ in (5).

Observe that, in the equivalent problem (4), the optimizing variables are reduced to $\left\{y_{l, c}, p_{l, c}\right\}, \forall l, c$. Consequently, the feasibility of the original problem (3) is now explicitly revealed by the constraint (4d), which implies that the interference power should not surpass the remaining power of the BS (i.e., $P_{B S}^{\max }-$ $\left.\sum_{c=1}^{C} \frac{\alpha_{c}}{g_{c}}\right)$.

Next, we investigate the objective in (4). For this purpose, let us rewrite $\tilde{r}_{l}$ as

$$
\tilde{r}_{l}=\sum_{c=1}^{C} y_{l, c} \log _{2}\left(1+w\left(p_{l, c}\right)\right),
$$

where $w\left(p_{l, c}\right)=d_{l, c} p_{l, c} /\left(e_{l, c}+f_{l, c} p_{l, c}\right)$. By checking the second-order derivative of $w\left(p_{l, c}\right)$

$$
w^{\prime \prime}\left(p_{l, c}\right)=-\frac{2 d_{l, c} e_{l, c} f_{l, c}}{\left(e_{l, c}+f_{l, c} p_{l, c}\right)^{3}} \leq 0,
$$

one can find that $w\left(p_{l, c}\right)$ is a concave function. Since the logarithm is an increasing and concave function, following the composition rule in [8], $\tilde{r}_{l}$ is also concave in $p_{l, c}$. Recalling that each $U_{l}\left(\tilde{r}_{l}\right)$ is an increasing and concave function of $\tilde{r}_{l}$, the objective in (4a) is thus concave in $p_{l, c}$.

Note that the problem (4) also includes another class of variable $y_{l, c}$. Towards achieving a tractable joint optimization, we 
temporarily relax $y_{l, c}$ to the interval $[0,1]$ and replace $p_{l, c}$ with a new variable $s_{l, c}=y_{l, c} p_{l, c}$. It is then not difficult to see that all constraints in (4) are jointly convex in $\left\{y_{l, c}, s_{l, c}\right\}$. More importantly, observe that $\tilde{r}_{l}\left(y_{l, c}, s_{l, c}\right)$ is a perspective function of $\tilde{r}_{l}\left(s_{l, c}\right)$ [9], and from the above property $\tilde{r}_{l}\left(s_{l, c}\right)$ is concave (since $\tilde{r}_{l}\left(p_{l, c}\right)$ is concave). Therefore, $\tilde{r}_{l}$ is jointly concave in $y_{l, c}$ and $s_{l, c}$, and (4) preserves convexity. Based on the above analysis, we analytically characterize the optimal power control and matching as follows.

Proposition 2: Suppose that D2D link $l$ reuses the RB of CU $c$. Then, the optimal power control for D2D link $l$ on the RB of $\mathrm{CU} c$ is given by

$$
p_{l, c}^{\star}=\left(\sqrt{\left(\frac{x_{l, c}^{(1)}}{2 x_{l, c}^{(0)}}\right)^{2}-\frac{x_{l, c}^{(2)}\left(\mu_{l}, \lambda\right)}{x_{l, c}^{(0)}}}-\frac{x_{l, c}^{(1)}}{2 x_{l, c}^{(0)}}\right)^{+},
$$

where $x_{l, c}^{(0)}=\left(d_{l, c}+f_{l, c}\right) f_{l, c}, x_{l, c}^{(1)}=\left(d_{l, c}+2 f_{l, c}\right) e_{l, c}$, $x_{l, c}^{(2)}\left(\mu_{l}, \lambda\right)=e_{l, c}^{2}-U_{l}^{\prime}\left(\tilde{r}_{l}\right) e_{l, c} d_{l, c} /\left(\ln 2\left(\mu_{l}+\lambda \alpha_{c} / g_{c} g_{l, c}^{I}\right)\right)$, $U_{l}^{\prime}\left(\tilde{r}_{l}\right)$ is the first derivative with respect to $\tilde{r}_{l}$, and $\boldsymbol{\mu} \succeq \mathbf{0}$, $\lambda \succeq \mathbf{0}$ are the Lagrange multipliers associated with the constraints $(4 \mathrm{c})$ and $(4 \mathrm{~d})$, respectively. The D2D link matched with the $\mathrm{RB}$ of $\mathrm{CU} c$ for a given power allocation is

$$
y_{l, c}^{\star}=1, l=\underset{1 \leq \hat{l} \leq L}{\operatorname{argmax}} H_{\hat{l}, c} ; y_{\hat{l}, c}^{\star}=0, \forall \hat{l} \neq l,
$$

where

$$
\begin{aligned}
H_{l, c}\left(p_{l, c}\right)= & U_{l}^{\prime}\left(\tilde{r}_{l}\right) \log _{2}\left(1+\frac{d_{l, c} p_{l, c}}{e_{l, c}+f_{l, c} p_{l, c}}\right) \\
& -\frac{U_{l}^{\prime}\left(\tilde{r}_{l}\right) d_{l, c} e_{l, c} p_{l, c}}{\ln 2\left(e_{l, c}+f_{l, c} p_{l, c}\right)\left[e_{l, c}+\left(d_{l, c}+f_{l, c}\right) p_{l, c}\right]} .
\end{aligned}
$$

Proof: The above properties are obtained by investigating the KKT conditions of (4). The details are omitted due to space limitations.

Remark 1: From Proposition 2, the optimal strategy of resuing the $c$ th CU's RB is to assign it to the D2D link with largest $H_{l, c}$. According to the definition (10), $H_{l, c}$ mainly depends on different channel gains, which are independent continuous-valued random variables. Consequently, the possibility of the event $H_{l, c}=H_{\tilde{l}, c}(l \neq \tilde{l})$ is zero in practice. Therefore, although $y_{l, c}$ is relaxed to $[0,1]$, the obtained matching schemes are still integer indications. This property is also verified by the numerical results in the next section.

Exploiting the above results, we then propose an iterative algorithm to jointly optimize the matching and power control of the D2D links and CUs, which is formally described in Algorithm 1. The basic idea of Algorithm 1 is to properly assign the available RBs to the D2D links via searching for the optimal $\left\{\lambda^{\star}, \boldsymbol{\mu}^{\star}\right\}$ by minimizing the dual function of (4). Algorithm 1 consists of two nested loops, which are responsible for updating the Lagrangian multipliers $\lambda$ and $\mu$, respectively. The optimal power allocation of the D2D links and D2D-CU matching are obtained from (8) and (9), respectively, while the Lagrangian multipliers $\lambda$ and $\boldsymbol{\mu}$ are updated according to the power constraints (4c) and (4d), respectively. Note that the step size $\theta_{j}$ in the Sub-Algorithm could use a diminishing rule such as $\theta_{j}=M /(M+j)$, where $M$ is a fixed integer.

\section{Algorithm 1 for Joint RB Assignment and Power Allocation}

Input: $P_{B S}^{\max } ; P_{l}^{\max }, \forall l ; R_{c}, g_{c} \forall c ; g_{l, c}, g_{l, c}^{I}, g_{c, l}^{I}, \forall c, l$.

Output: $p_{c}^{\star}, \forall c ; y_{l, c}^{\star}, p_{l, c}^{\star}, \forall l, c$.

Initialization: set precision $\epsilon ; l_{a}=0, l_{b}=\lambda_{\max } ; k=1$,

$\lambda_{k}=\frac{l_{a}+l_{b}}{2}$; let $P^{\text {sur }} \triangleq P_{B S}^{\max }-\sum_{c=1}^{C} \frac{\alpha_{c}}{g_{c}}$.

While $\left|l_{a}-l_{b}\right|>\epsilon$

1. Find the optimal $\boldsymbol{\mu}^{\star}$ and $p_{l, c}^{\star}, \forall l, c$ for given $\lambda_{k}$ via Sub-Algorithm below.

2. $P^{s u r^{\prime}}=\sum_{c=1}^{C} \sum_{l=1}^{L} \frac{\alpha_{c}}{g_{c}} g_{l, c}^{I} y_{l, c} p_{l, c}$.

3. If $P^{s u r^{\prime}}<P^{s u r}, l_{b}=\lambda_{k}$, otherwise, $l_{a}=\lambda_{k}$.

4. Update $\lambda_{k+1}=\left(l_{a}+l_{b}\right) / 2, k=k+1$.

End While

Sub-Algorithm:

Initialize $j=1$ and $\boldsymbol{\mu}_{j}$ with some positive vector.

Repeat

a) Compute $p_{l, c}^{\star}, \forall l, c$ with given $\lambda_{k}, \boldsymbol{\mu}_{j}$ via (8).

b) Compute $H_{l, c}$ for any $l, c$ via (10).

c) Match D2D link $l$ to $\mathrm{CU} c$ according to (9).

d) Update $\mu_{l, j+1}, \forall l$ as

$$
\mu_{l, j+1}=\left[\mu_{l, j}-\theta_{j}\left(P_{l}^{\max }-\sum_{c=1}^{C} y_{l, c} p_{l, c}\right)\right]_{0}^{\mu_{l}^{\max }} .
$$

e) $j=j+1$.

Until $\left|\mu_{l, j}-\mu_{l, j-1}\right|<\epsilon$ for all $l$.

Finally, we calculate $p_{c}^{\star}=\frac{\alpha_{c}}{g_{c}}\left(1+g_{l, c}^{I} p_{l, c}^{\star}\right)$, for $y_{l, c}^{\star}=1$.

The proposed algorithm requires upper bounds for the optimal Lagrangian multipliers $\lambda$ and $\boldsymbol{\mu}$. Although one may use very large values, properly chosen bounds will lead to faster convergence. The following result provides such bounds for $\lambda$ and $\mu$.

Lemma 1: The optimal Lagrangian multipliers $\lambda^{\star}$ and $\mu_{l}^{\star}$ are in the intervals $\left[0, \lambda^{\max }\right]$ and $\left[0, \mu_{l}^{\max }\right]$, where

$$
\lambda^{m a x}=\max _{1 \leq l \leq L, 1 \leq c \leq C} d_{l, c} g_{c} /\left(\ln 2 e_{l, c} g_{l, c}^{I} \alpha_{c}\right)
$$

and

$$
\mu_{l}^{\max }=\max _{1 \leq c \leq C} d_{l, c} / \ln 2 e_{l, c}, \forall l,
$$

respectively.

Proof: From (8) and (4d), there is at least one $p_{l_{c}}^{\star} \geq 0$ in the $c$ th RB if and only if $e_{l, c}-d_{l, c} /\left(\ln 2\left(\lambda \alpha_{c} / g_{c}+\mu_{l}\right)\right)<0$ for any $l$ and $c$. Let $\mu_{l}=0$ or $\lambda=0$ respectively, we then obtain the $\lambda^{\max }$ and $\mu_{l}^{\max }$. 


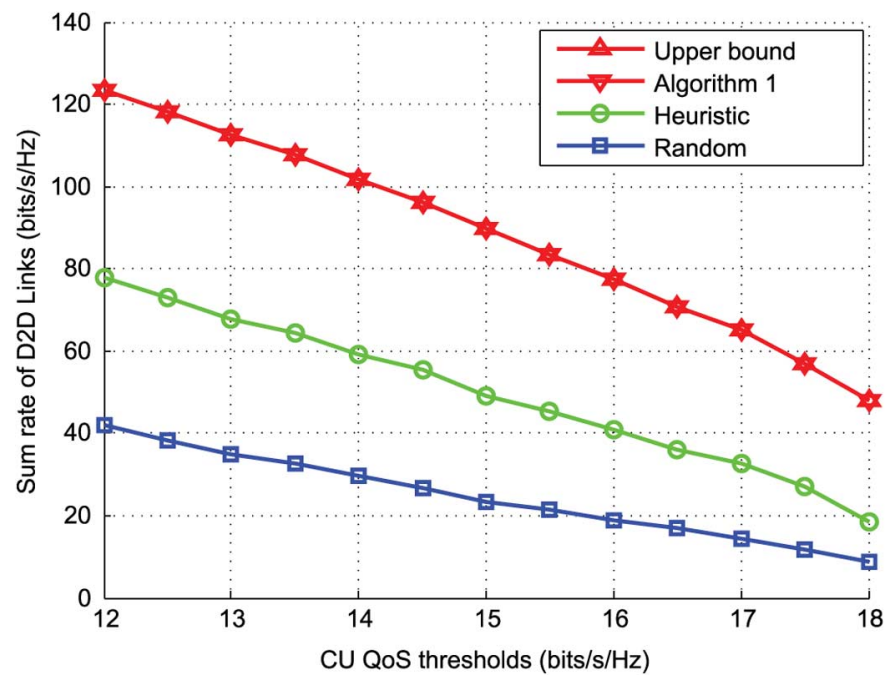

Fig. 2. Sum rate of the D2D links for different CU QoS thresholds with 15 D2D links and 10 CUs.

\section{Numerical Results}

In this section, we present some numerical results to evaluate the performance of our proposed algorithm. In addition to the optimal approach in (8) and (9), the methods compared include the one in [6] and a random reuse scheme, which are labelled as 'Heuristic' and 'Random', respectively. To evaluate the optimality of Algorithm 1, we also illustrate an upper bound given by the dual problem of (4). The simulation parameters mainly follow from the Urban Macro (UMa) model for LTE-Advanced in [10], including the cell radius $(500 \mathrm{~m})$, pathloss model for channels from the BS to the CUs or D2D RXs, thermal noise level $(-174 \mathrm{dBm} / \mathrm{Hz}), \mathrm{RB}$ bandwidth $(15 \mathrm{KHz})$, power budgets of BS and D2D TX (46dBm and 20dBm, respectively), etc. The channels from D2D TX to D2D RX and from D2D TX to the CUs follow an exponential path-loss model with exponent of 3.5. The CUs and D2D links are uniformly distributed within the range of the cell and the distances between D2D TX and $\mathrm{RX}$ are set to be $20 \mathrm{~m}$. In the following numerical examples, we use the sum rate of the D2D links as our utility function.

In Fig. 2, we present the sum rate of the D2D links for different CU QoS requirements. As the QoS threshold increases, the performance of all methods clearly deteriorates. One can observe that Algorithm 1 has a huge advantage over the other methods, achieving nearly $50 \%-100 \%$ better performance than the other methods. In Fig. 3, we show the D2D sum rate for different numbers of CUs. As the number of CUs increases, the D2D links have more cellular resources to reuse and this leads to higher sum rates. On the other hand, since the BS has limited power, it becomes harder to satisfy the CU QoS requirement for more CUs, so the D2D reuse performance tends to saturate or even decline. Note that in both figures, the performance gap between Algorithm 1 and the upper bound is negligible, which implies that Algorithm 1 achieves the optimal RR solution.

\section{CONCLUSION}

In this letter, we studied downlink reuse strategies for multiple underlay D2D links with multiple CUs. We formulated a network utility maximization problem for D2D communications with the CUs protected by the QoS constraints. We

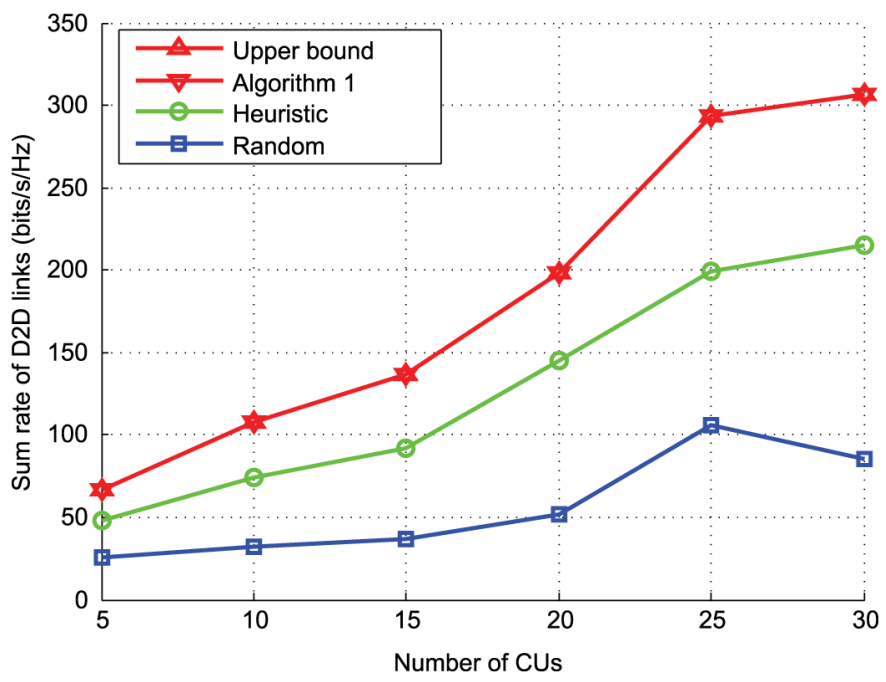

Fig. 3. Sum rate of the D2D links versus the number of CUs with 15 D2D links and the CU QoS threshold at $15 \mathrm{bits} / \mathrm{s} / \mathrm{Hz}$.

first transformed the problem into a more tractable form by revealing the CUs' optimal power requirements that fulfill the QoS provisions. Under this equivalent transformation, the jointly nonconvex power coordination of the CUs and D2D links can be simplified to a convex power control problem associated with the D2D links. Then we incorporated the relaxed D2D-CU matching variables into the problem and analytically characterized the optimal resource reuse through convex optimization techniques. Based on the analytical results, we proposed an efficient algorithm to jointly optimize the D2D-CU matching and power control. The effect and optimality of the proposed method were verified by several numerical examples.

\section{REFERENCES}

[1] D. H. Zhu, A. L. Swindlehurst, S. A. A. Fakoorian, W. Xu, and C. M. Zhao, "Device-to-device communications: The physical layer security advantage," in IEEE Int. Conf. Acoustics, Speech, and Signal Processing (ICCASP), 2014.

[2] P. Phunchongharn, E. Hossain, and D. I. Kim, "Resource allocation for device-to-device communications underlaying LTE-advanced networks," IEEE Wireless Commun., vol. 20, no. 4, pp. 91-100, Aug. 2013.

[3] C.-H. Yu, K. Doppler, C. B. Ribeiro, and O. Tirkkonen, "Resource sharing optimization for device-to-device communication underlaying cellular networks," IEEE Trans. Wireless Commun., vol. 10, no. 8, pp. 2752-2763, Aug. 2011

[4] H. Min, J. Lee, S. Park, and D. Hong, "Capacity enhancement using an interference limited area for device-to-device uplink underlaying cellular networks," IEEE Trans. Wireless Commun., vol. 10, no. 12, pp. 3995-4000, Dec. 2011.

[5] D. Feng, L. Lu, Y. Yuan-Wu, G. Y. Li, G. Feng, and S. Li, "Device-todevice communications underlaying cellular networks," IEEE Trans. Commun., vol. 61, no. 8, pp. 3541-3551, Aug. 2013.

[6] M. Zulhasnine, C. Huang, and A. Srinivasan, "Efficient resource allocation for device-to-device communication underlaying LTE network," in Proc. IEEE WiMob 2010, Oct. 2010, pp. 368-375.

[7] F. Kelly, A. Maulloo, and D. Tan, "Rate control in communication networks: Shadow prices, proportional fairness and stability," J. Operational Research Society, vol. 49, pp. 237-252, 1998.

[8] S. Boyd and L. Vandenberghe, Convex Optimization. Cambridge, U. K.: Cambridge University Press, 2004.

[9] C. Y. Wong, R. S. Cheng, K. B. Lataief, and R. D. Murch, "Multiuser OFDM with adaptive subcarrier, bit, and power allocation," IEEE J. Sel. Areas Commun., vol. 17, no. 10, pp. 1747-1758, Oct. 1999.

[10] Guidelines for evaluation of radio interface technologies for IMT-Advanced Rep. M.2135-0, 2008 